\title{
Rigorous Coupled-Wave Approach for Sandwich Gratings
}

\author{
Xuehui Xiong1, Ping Lu² \\ ${ }^{1}$ School of Physics and Information Engineering, Jianghan University, Wuhan, China \\ ${ }^{2}$ School of Optical and Electronic Information, Huazhong University of Science and Technology, Wuhan, China \\ Email: xuehuixiong@163.com
}

Received 6 July 2016; accepted 6 August 2016; published 9 August 2016

Copyright (C) 2016 by authors and Scientific Research Publishing Inc.

This work is licensed under the Creative Commons Attribution International License (CC BY).

http://creativecommons.org/licenses/by/4.0/

(c) (i) Open Access

\begin{abstract}
Rigorous Coupled-Wave Approach (RCWA) has been used successfully and accurately to study simple grating structures, such as one-layer gratings, one-whole gratings. In this paper, RCWA is expanded to solve Sandwich gratings (SG), which is composed of two identical planar dielectric gratings adjoined by thin metallic or dielectric film. The electromagnetic analytic expressions for each layer of SG structure are given and rigorous coupled-wave equations are deduced. The numerical investigations for the diffraction spectra of SG by our theoretical and computer programs are in good agreement with the results of classical RCWA in the condition when a Sandwish grating is simplified to a classical one-layer grating. The calculations by our programs of another condition when a Sandwish grating is degenerated to a classical single planar structure also conform to the results of classical electromagnetic theory. The research results above indicate that the extended theoretical formula has backwards compatibility and is self-consistent with the classical theory.
\end{abstract}

\section{Keywords}

Rigorous Coupled-Wave Approach, Sandwich Gratings, Self-Consistent

\section{Introduction}

Over the past 30 years RCWA formulated by Moharam and Gaylord [1]-[5] has been used successfully and accurately to analyze periodic structures including holographic gratings [1] [2] and arbitrary profiled dielectric or metallic surface-relief gratings [3] [6]-[8]. RCWA is almost used to study relatively simple structure, such as one-layer gratings [1]-[5] and one-whole gratings [6]-[13] which have arbitrary profiled surface-relief on both of top and bottom of monolithic materials. Owing to its complexity and difficulty, RCWA is seldom used to study 
multi-layers grating.

In this paper, RCWA is adopted to solve sandwich gratings (SG) structure, which is composed of two identical planar dielectric gratings adjoined by thin metallic or dielectric film. The electromagnetic analytic expressions for each layer of SG structure are given and rigorous coupled-wave equations are deduced. To verify the theory presented in the paper, the proposed RCWA for SG and classic electromagnetic theory are respectively used to research two degenerative SG structures, namely classical single grating and classical single planar structure. The results indicate that RCWA for SG has backwards compatibility and is self-consistent with the classical theory.

\section{The Sandwich Grating Structure and Theoretical Formulas}

A schematic diagram of the proposed Sandwich grating structure is shown in Figure 1. The configuration consists of two identical planar sinusoidal dielectric gratings of thickness $d$ adjoined by continuous thin silver or dielectric film of thickness $h$. The lossless planar dielectric grating [1] [2] is characterized by a periodical medium. The relative permittivity can be depicted

$$
\varepsilon_{2}(x, z)=\varepsilon_{4}(x, z)=\varepsilon_{\text {avg }}+\Delta \varepsilon \cos [K(x \sin \phi+z \cos \phi)]
$$

where $\varepsilon_{a v g}$ is the average permittivity and $\Delta \varepsilon$ is the amplitude of the sinusoidal permittivity. $\phi$ is the grating slant angle and $K=2 \pi / \Lambda$, here $\Lambda$ is the grating period. The permittivity in the region I $(z<0)$ is $\varepsilon_{1}$ and the ones in the region $\mathrm{V}(z>2 d+h)$ is $\varepsilon_{5}$. While the permittivity of Ag film in the region III is $\varepsilon_{3}$. The complex permittivity of metallic films is described by the Drude model

$$
\varepsilon_{3}(\omega)=1-\frac{\omega_{p}^{2}}{\omega^{2}+j \gamma \omega}
$$

where $\omega_{p}=1.37 \times 10^{16} \mathrm{rad} / \mathrm{s}$ is the plasma frequency for $\mathrm{Ag}$ and $\gamma=7.29 \times 10^{13} \mathrm{rad} / \mathrm{s}$ is the collision frequency for Ag [14], $j=\sqrt{-1}$.

For E-mode polarization (the electric field is in the plane of incidence), the magnetic field is solely in the $y$ direction. According to rigorous coupled-wave analysis theory (RCWA) [1]-[5], the normalized magnetic fields in each region may be expressed as:

$$
\text { Region I } H_{1 y}=\mathrm{e}^{-j\left(k_{x 0} x+k_{170} z\right)}+\sum_{i} R_{1 i} \mathrm{e}^{-j\left(k_{x i} x-k_{17 i} z\right)}
$$

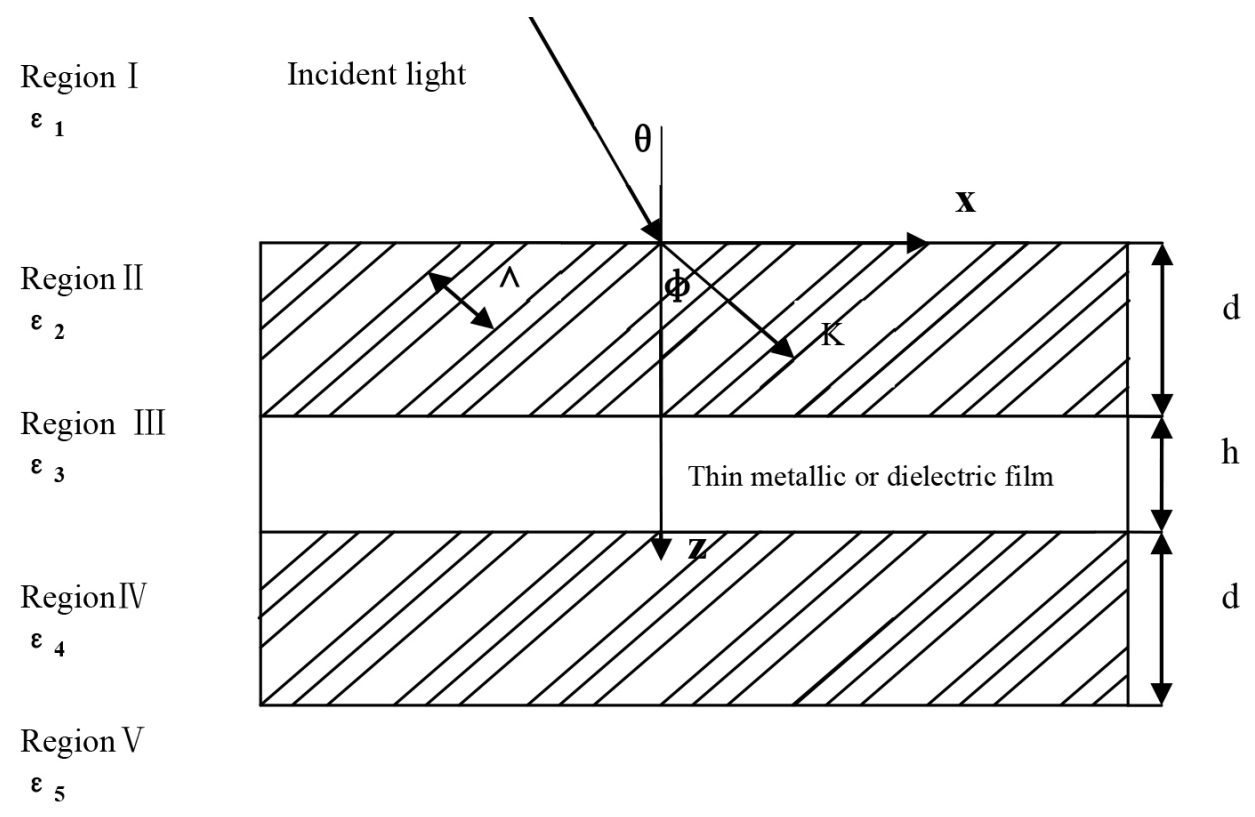

Figure 1. Schematic diagram of Sandwish grating structure. 


$$
\begin{gathered}
\text { Region II } H_{2 y}=\sum_{i} U_{2 i}(z) \mathrm{e}^{-j\left(k_{x i} x+k_{2 z i} z\right)} \\
\text { Region III } H_{3 y}=\sum_{i} T_{3 i} \mathrm{e}^{-j\left(k_{k i} x+k_{3 i i}(z-d-h)\right)}+\sum_{i} R_{3 i} \mathrm{e}^{-j\left(k_{x i} x-k_{3 i}(z-d-h)\right)} \\
\text { Region IV } H_{4 y}=\sum_{i} U_{4 i}(z-d-h) \mathrm{e}^{-j\left(k_{x i} x+k_{4 i i}(z-d-h)\right)} \\
\text { Region V } H_{5 y}=\sum_{i} T_{5 i} \mathrm{e}^{-j\left(k_{x i} x+k_{5 z i}(z-2 d-h)\right)}
\end{gathered}
$$

And $U_{2 i}(z), U_{4 i}(z)$ are the space harmonic magnetic-field amplitudes and satisfy coupled-wave equations in grating regions. The solutions for $U_{2 i}(z), U_{4 i}(z)$ are referenced from the Ref. [2] and may be expressed as: $U_{2 i}(z)=\sum_{n=1}^{2 m} C_{n} \omega_{i m} \mathrm{e}^{\lambda_{m} z}, U_{4 i}(z)=\sum_{n=2 m+1}^{4 m} C_{n} \omega_{i m} \mathrm{e}^{\lambda_{m^{z}}}$ where $\lambda_{m}$ and $\omega_{i m}$ are the eigenvalues and eigenvectors. The second grating in Region IV is the same modulated as the first grating, so their eigenvalues and eigenvectors are also the same. But owing to the different boundary conditions of tangential electric and magnetic fields, the coefficients $C_{n}$ are different. On the other hand, the normalized wave amplitudes of the thin connected region (Region III) are determined by the interactions between the forward-diffraction of the first grating and backward-diffraction of the second grating.

The symbols used in Equations (3)-(7) are as follows:

$$
\begin{gathered}
k_{x i}=2 \pi \sqrt{\varepsilon_{1}} / \lambda \sin \theta-i K \sin \phi \\
k_{l z i}=\sqrt{\left(k_{l}^{2}-k_{x i}^{2}\right)}, k_{l}=2 \pi \sqrt{\varepsilon_{l}} / \lambda, l=1,3,5 \\
k_{\xi z i}=\sqrt{\left(k_{\xi}^{2}-k_{x 0}^{2}\right)}-i K \cos \phi, k_{\xi}=2 \pi \sqrt{\varepsilon_{\xi}} / \lambda, \xi=2,4
\end{gathered}
$$

where $i$ is the space-harmonic index in grating Regions II and IV (analogous to the diffractive order index in Regions I, III and V), $\theta$ is the angle of incidence, $\lambda$ is the free-space wavelength. $R_{l i}, T_{l i}$ are the normalized amplitude of the ith reflected and transmitted wave of Region I, Region III or Region V.

The electromagnetic boundary conditions require that the tangential components of the electric field and the magnetic field must be continuous across planes $z=0, z=d, z=d+h$ and $z=2 d+h$. The boundary conditions for tangential magnetic field $\left(H_{y}\right)$ are respectively

$$
\begin{gathered}
U_{2 i}(0)=\delta_{i 0}+R_{1 i} \\
U_{2 i}(d) \mathrm{e}^{-j k_{2 z i} d}=T_{3 i} \mathrm{e}^{j k_{3 i} h}+R_{3 i} \mathrm{e}^{-j k_{3 z i} h} \\
U_{4 i}(0)=T_{3 i}+R_{3 i} \\
U_{4 i}(d) \mathrm{e}^{-j k_{4 i i} d}=T_{5 i}
\end{gathered}
$$

The tangential electric field $E_{x}$ may be obtained from the Maxwell curl equation $\nabla \times \boldsymbol{H}=j \omega \varepsilon_{0} \varepsilon_{r} \boldsymbol{E}$. The result is $E_{x}=j /\left(\omega \varepsilon_{0} \varepsilon_{r}(x, z)\right) \partial H_{y} / \partial z$ and boundary conditions for tangential electric field $\left(E_{x}\right)$ are respectively

$$
\begin{aligned}
& \frac{1}{\varepsilon_{1}}\left(R_{1 i}-\delta_{i 0}\right)\left(j k_{1 z i}\right)=\sum_{l} \varepsilon_{2}^{-1}(l)\left(U_{2(i-l)}^{\prime}(0)+U_{2(i-l)}(0)\left(-j k_{2 z(i-l)}\right)\right) \\
& \quad \frac{1}{\varepsilon_{3}}\left(T_{3 i}\left(-j k_{3 z i}\right) \mathrm{e}^{j k_{3 z i} h}+R_{3 i}\left(j k_{3 z i}\right) \mathrm{e}^{-j k_{3 z i} h}\right) \\
& \quad=\sum_{l} \varepsilon_{2}^{-1}(l) \mathrm{e}^{-j k_{2 z i} d}\left(U_{2(i-l)}^{\prime}(d)+U_{2(i-l)}(d)\left(-j k_{2 z(i-l)}\right)\right)
\end{aligned}
$$




$$
\begin{gathered}
\frac{1}{\varepsilon_{3}}\left(T_{3 i}\left(-j k_{3 z i}\right)+R_{3 i}\left(j k_{3 z i}\right)\right)=\sum_{l} \varepsilon_{4}^{-1}(l)\left(U_{4(i-l)}^{\prime}(0)+U_{4(i-l)}(0)\left(-j k_{4 z(i-l)}\right)\right) \\
\frac{1}{\varepsilon_{5}}\left(T_{5 i}\left(-j k_{5 z i}\right)\right)=\sum_{l} \varepsilon_{4}^{-1}(l) \mathrm{e}^{-j k_{4 z i} d}\left(U_{4(i-l)}^{\prime}(d)+U_{4(i-l)}(d)\left(-j k_{4 z(i-l)}\right)\right)
\end{gathered}
$$

where $U^{\prime}(z)=\mathrm{d} U(z) / \mathrm{d} z$.

If $\mathrm{N}$ values of $i$ are retained in the analysis, there will be $4 \mathrm{~N}$ unknown values of $C_{n}$ and they will be determined from the boundary conditions. All the $R_{l i}, T_{l i}$ may then be calculated.

The backward-wave diffraction efficiencies (Region I) are

$$
D E_{1 i}=\left|R_{1 i}\right|^{2} \operatorname{Re}\left(k_{1 z i} / k_{1 z 0}\right)
$$

The forward-wave diffraction efficiencies (Region V) are

$$
D E_{5 i}=\left|T_{5 i}\right|^{2} \operatorname{Re}\left(k_{5 z i} \times \varepsilon_{1} /\left(\varepsilon_{5} \times k_{1 z 0}\right)\right)
$$

\section{Numerical Calculations and Discussions}

In order to verify the deduced formulas above, the reflection and transmission characteristics of Sandwich gratings connected by thin silver film are studied in the condition of grating thickness $d=0$ at normal incidence. The other parameters are as follows, $\varepsilon_{1}=\varepsilon_{5}=1.33^{2}, h=40 \mathrm{~nm}$. The five calculated regions shown in Figure 2(a) are degenerated into three regions shown in Figure 2(b). The proposed RCWA for SG and classic electromagnetic theory are respectively used to solve for the reflection and transmission characteristics. The efficiencies of results given by RCWA in the paper and classical theory are both shown in Figure 3. The discrepancy magnitude between two methods is only $10^{-5}$ shown in Figure 4, which proves our formulas and computer program codes to be true. Our work backwards contains the results of the classical Fresnel formulas of three regions.

Furthermore, when the connection layer of thin metallic film is absence, namely $h=0 \mathrm{~nm}$, the proposed Sandwich grating is simplified into an ordinary one-layer grating. The unslant grating has $400 \mathrm{~nm}$ grating period and $100 \mathrm{~nm}$ thickness, and its average permittivity is 2.25 with the modulation 0.33 . The thickness of the connection layer of thin Ag film is zero. On this condition, the two same gratings are combined to be one whole thick grating of $200 \mathrm{~nm}$, shown in Figure 5(b). Suppose the grating in water, thus $\varepsilon_{1}=\varepsilon_{5}=1.33^{2}$.

The efficiencies of reflection and transmission of single layer gratings calculated by our theory and by classical Rigorous Coupled-wave Approach are both shown in Figure 6. The results are nearly the same.

From the above discussed, the correctness and efficiencies of RCWA for SG are verified. The theory given in the paper has backwards compatibility and is self-consistent with the classical theory.

\section{Conclusion}

Rigorous coupled-wave approach for SG is proposed in the paper. The proposed RCWA for SG and classic electromagnetic theory are respectively used to study two degenerative SG structures, and the reflection and

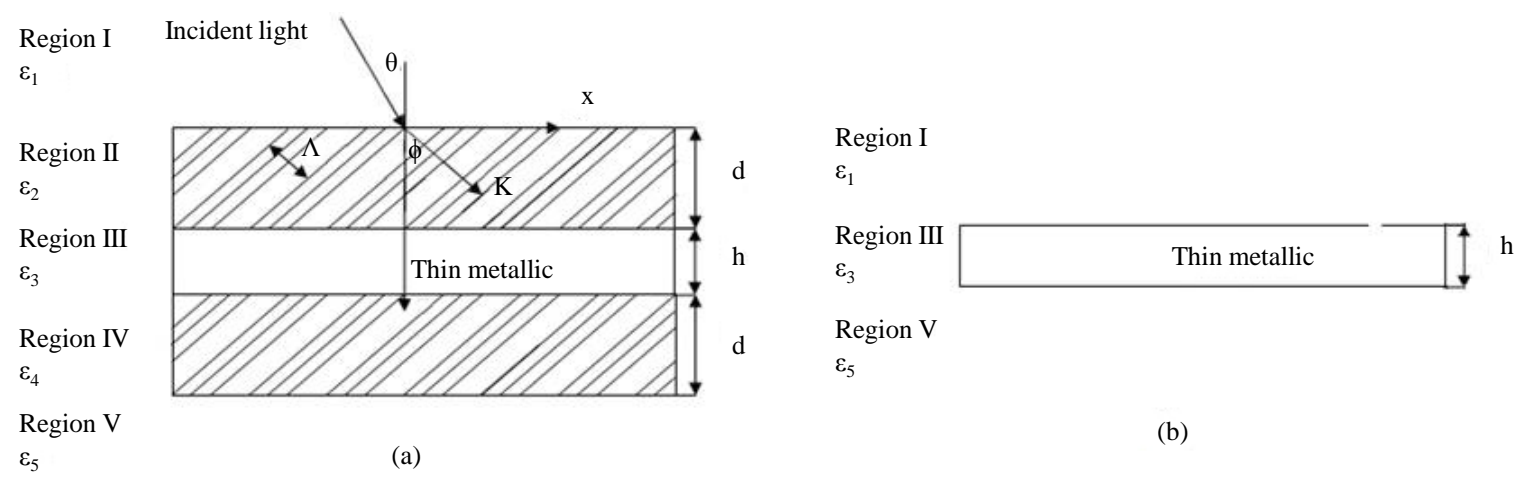

Figure 2. (a) Sandwish grating structure; (b) classical planar structure. 


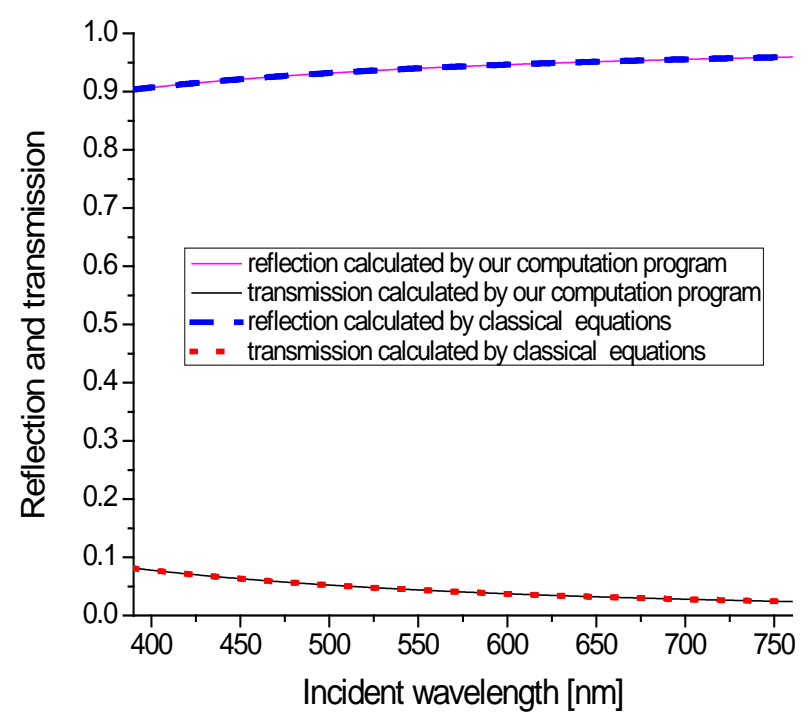

Figure 3. Reflection and transmission at normal incidence with $d=0, \varepsilon_{1}=\varepsilon_{5}=1.33^{2}, h=40 \mathrm{~nm}$.

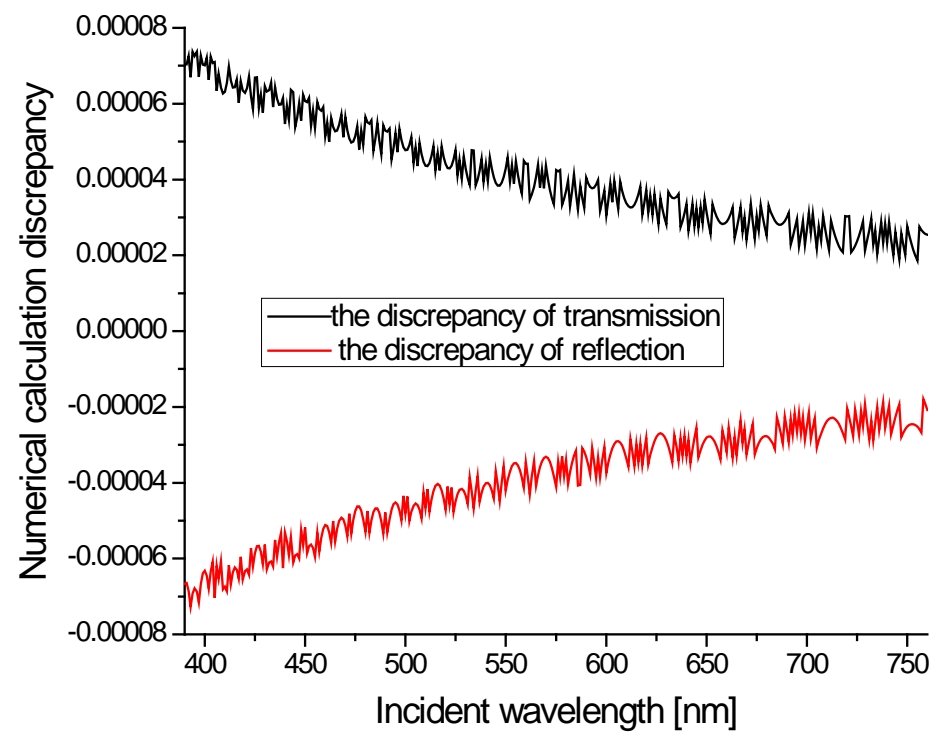

Figure 4. Calculation discrepancy between our program and Fresnel equations.

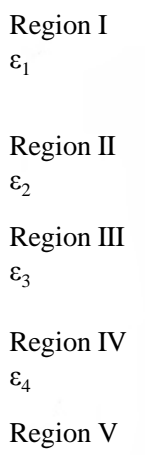
$\varepsilon_{5}$

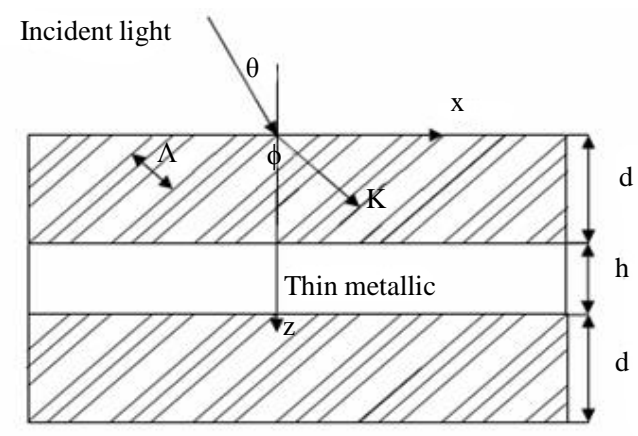

(a)
Incident light

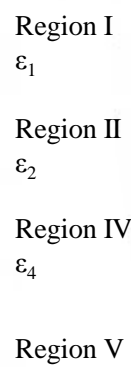

$\varepsilon_{5}$

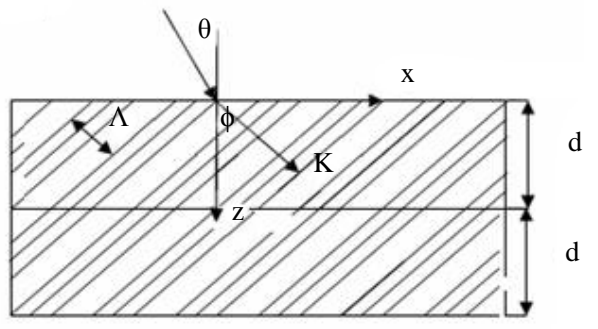

(b)

Figure 5. (a) Sandwish grating; (b) Combined into single layer grating. 


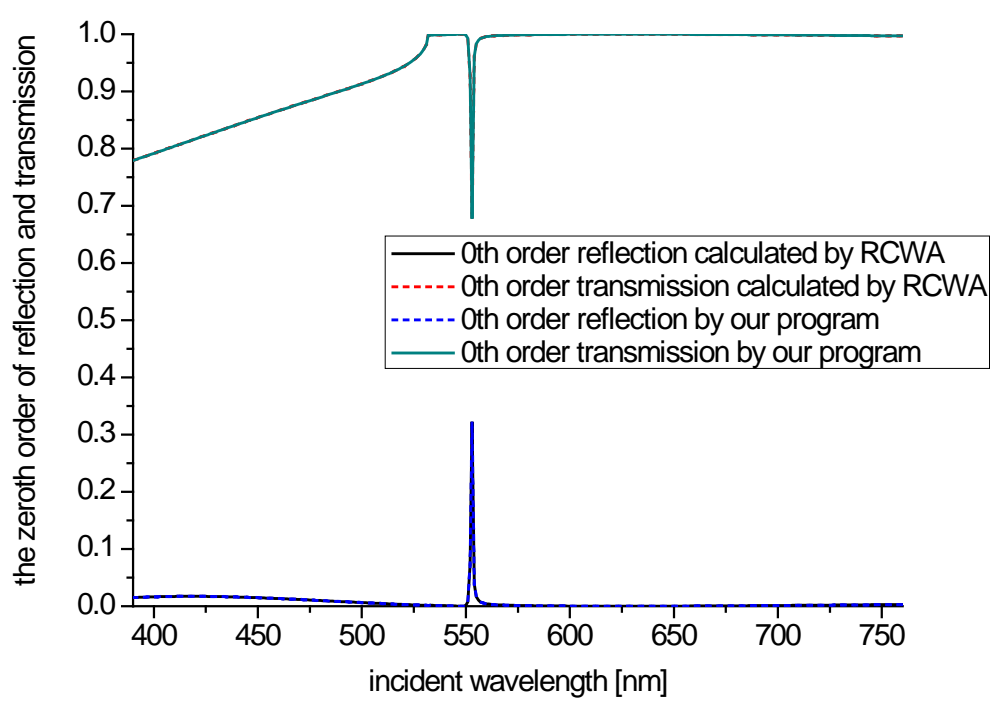

Figure 6. Reflection and transmission at normal incidence with $d=100 \mathrm{~nm}$, $\varepsilon_{1}=\varepsilon_{5}=1.33^{2}, h=0$.

transmission spectra are almost the same. The results indicate that RCWA for SG has backwards compatibility and is self-consistent with the classical theory. The theoretical formula and computer codes lay the foundations for investigation of properties of the novel Sandwich grating and exploitation of nano-photonics devices.

\section{Acknowledgements}

The author appreciates the support from National Natural Science Foundation of China under Grant No. 61275083.

\section{References}

[1] Moharam, M.G. and Gaylord, T.K. (1981) Rigorous Coupled-Wave Analysis of Planar-Grating Diffraction. Journal of the Optical Society of America, 71, 811-818. http://dx.doi.org/10.1364/JOSA.71.000811

[2] Moharam, M.G. and Gaylord, T.K. (1983) Rigorous Coupled-Wave Analysis of Grating Diffraction-E-Mode Polarization and Losses. Journal of the Optical Society of America, 73, 451-455. http://dx.doi.org/10.1364/JOSA.73.000451

[3] Moharam, M.G. and Gaylord, T.K. (1986) Rigorous Coupled-Wave Analysis of Metallic Surface-Relief Gratings. Journal of the Optical Society of America A, 3, 1780-1787. http://dx.doi.org/10.1364/JOSAA.3.001780

[4] Lalanne, P. and Michael Morries, G. (1996) Highly Improved Convergence of the Coupled-Wave Method for TM Polarization. Journal of the Optical Society of America A, 13, 779-784. http://dx.doi.org/10.1364/JOSAA.13.000779

[5] Lalanne, P. (1997) Improved Formulation of the Coupled-Wave Method for Two-Dimensional Gratings. Journal of the Optical Society of America A, 14, 1592-1598. http://dx.doi.org/10.1364/JOSAA.14.001592

[6] Porto, J.A., García-Vidal, F.J. and Pendry, J.B. (1999) Transmission Resonances on Metallic Gratings with Very Narrow Slits. Physical Review Letters, 83, 2845-2848. http://dx.doi.org/10.1103/PhysRevLett.83.2845

[7] Bonod, N., Popov, E. and McPhedran, R.C. (2008) Increased Surface Plasmon Resonance Sensitivity with the Use of Double Fourier Harmonic Gratings. Optics Express, 16, 11691-11702. http://dx.doi.org/10.1364/oe.16.011691

[8] Gérard, D., Salomon, L., de Fornel, F. and Zayats, A.V. (2004) Ridge-Enhanced Optical Transmission through a Continuous Metal Film. Physical Review B, 69, 113405. http://dx.doi.org/10.1103/physrevb.69.113405

[9] Fong, K.-Y. and Huia, P.M. (2006) Coupling of Waveguide and Surface Modes in Enhanced Transmission through Stacking Gratings. Applied Physics Letters, 89, 091101. http://dx.doi.org/10.1063/1.2338796

[10] Ebbesen, T.W., Lezec, H.J., Ghaemi, H.F., Thio, T. and Wolff, P.A. (1998) Extraordinary Optical Transmission through Sub-Wavelength Hole Arrays. Nature, 391, 667-669. http://dx.doi.org/10.1038/35570

[11] Koerkamp, K.J.K., Enoch, S., Segerink, F.B., van Hulst, N.F. and Kuipers, L. (2004) Strong Influence of Hole Shape on Extraordinary Transmission through Periodic Arrays of Subwavelength Holes. Physical Review Letters, 92, 183901. http://dx.doi.org/10.1103/PhysRevLett.92.183901 
[12] Shin, H., Catrysse, P.B. and Fan, S. (2005) Effect of the Plasmonic Dispersion Relation on the Transmission Properties of Subwavelength Cylindrical Holes. Physical Review B, 72, 085436. http://dx.doi.org/10.1103/physrevb.72.085436

[13] Lu, H., Li, J., Ong, H.C. and Wan, J.T.K. (2008) Surface Plasmon Resonance in Two-Dimensional Nanobottle Arrays. Optics Express, 16, 10294-10302. http://dx.doi.org/10.1364/OE.16.010294

[14] Rakić, A.D., Djurišic, A.B., Elazar, J.M. and Majewski, M.L. (1998) Optical Properties of Metallic Films for VerticalCavity Optoelectronic Devices. Applied Optics, 37, 5271-5283. http://dx.doi.org/10.1364/AO.37.005271

\section{Submit or recommend next manuscript to SCIRP and we will provide best service for you:}

Accepting pre-submission inquiries through Email, Facebook, LinkedIn, Twitter, etc. A wide selection of journals (inclusive of 9 subjects, more than 200 journals)

Providing 24-hour high-quality service

User-friendly online submission system

Fair and swift peer-review system

Efficient typesetting and proofreading procedure

Display of the result of downloads and visits, as well as the number of cited articles

Maximum dissemination of your research work

Submit your manuscript at: http://papersubmission.scirp.org/ 